\title{
Spatiotemporal Focusing Dynamics of Intense Supercontinuum THz Pulses
}

\author{
Clemens Ruchert, ${ }^{1, *}$ Carlo Vicario, ${ }^{1}$ and Christoph P. Hauri ${ }^{1,2, \dagger}$ \\ ${ }^{1}$ Paul Scherrer Institute, SwissFEL, 5232 Villigen PSI, Switzerland \\ ${ }^{2}$ Ecole Polytechnique Federale de Lausanne, 1015 Lausanne, Switzerland \\ (Received 29 October 2012; published 22 March 2013)
}

\begin{abstract}
High-field terahertz (THz) single-cycle pulses with $1.5 \mathrm{MV} / \mathrm{cm}$ are generated by optical rectification in the stilbazolium salt crystal 4- $N, N$-dimethylamino-4'- $N$ '-methyl-stilbazolium 2,4,6trimethylbenzenesulfonate. We show experimentally that the generated $\mathrm{THz}$ transient carrying 5 octaves $(0.15$ to $5.5 \mathrm{THz})$ undergoes a complex time-frequency evolution when tightly focused, and we present a model based on three independent oscillating dipoles capable to describe this anomalous field evolution. Finally, we present a method to control the absolute phase of such supercontinuum $\mathrm{THz}$ pulses as an essential tool for future field-sensitive investigations.
\end{abstract}

The pursuit of high-field $(>\mathrm{MV} / \mathrm{cm})$ single-cycle and subcycle terahertz $(\mathrm{THz})$ pulses is inspired by the exploration of nonlinear field-induced phenomena in various areas such as accelerator physics [1], attosecond spectroscopy [2], and condensed matter physics [3]. Initiation of motion in solids (e.g., phonon modes, spin waves) [4] by an intense single-cycle terahertz pulse represent a new class of experiments to control dynamics by directly accessing the $\mathrm{THz}$ active port in absence of photoionization. The realization of highest field in the THz gap $(0.1-10 \mathrm{THz})$ well beyond the $\mathrm{MV} / \mathrm{cm}$ barrier has remained challenging, also due to beam aberration which hinders focusing to diffraction limited spot size [5,6]. For field-sensitive phenomena [7], the knowledge and ultimately the control of field strength, polarity, absolute and geometrical phase, instantaneous frequency, and the (non)linear evolution thereof across the focal region is of fundamental interest. The evolution of these parameters across the focal region has experimentally not been rigorously characterized for single-cycle, high-field $\mathrm{THz}$ pulses carrying a multioctave spanning spectrum.

In this Letter, we first present strong transients covering 5 octaves in the $\mathrm{THz}$ frequency range $(0.15-5.5 \mathrm{THz})$ generated by optical rectification in the organic crystal 4- $N, N$-dimethylamino-4'- $N$ '-methyl-stilbazolium 2,4,6trimethylbenzenesulfonate (DSTMS). We demonstrate transients with field strength of $1.5 \mathrm{MV} / \mathrm{cm}$ within one optical cycle in a close to diffraction limited spot size, thanks to the excellent $\mathrm{THz}$ focusing properties. We secondly investigate the complex propagation of this high-field $\mathrm{THz}$ transient through the focus (i.e., interaction zone for high-field $\mathrm{THz}$ experiments) and study the complex evolution of the field shape in the time and frequency domain. The evolution of the electric field across the focal region unveils not only the frequency-dependent polarity reversal due to the geometrical Gouy phase shift but also a distinct blueshift and time varying instantaneous frequency. These intrapulse variations are visualized by Wigner-Ville distributions (WVDs) based on direct measurements of the electric field along the focus. The experimental data are confirmed by the presented numerical model which is capable to describe the propagation of focused multioctave pulses across the focus. Finally, a concept is presented for controlling the absolute phase of such ultrabroadband transients in order to realize the most asymmetric $\mathrm{THz}$ field strength at the waist, with an absolute phase equal to zero.

We employ $\mathrm{THz}$ generation by optical rectification (OR) in the recently developed large-size organic crystal DSTMS [8]. This crystalline structure consists of a positively charged stilbazolium chromophore with a counter anion forcing crystalline noncentrosymmetric packing. Low $\mathrm{THz}$ absorption makes it superior to other organic rectifiers [9]. The large OR crystal (9 $\mathrm{mm}$ clear aperture, $480 \mu \mathrm{m}$ thick) is pumped with the collimated output of an optical parametric amplifier $(E=2 \mathrm{~mJ}, \quad I=$ $170 \mathrm{GW} / \mathrm{cm}^{2}, f_{\text {rep }}=100 \mathrm{~Hz}, \tau=70 \mathrm{fs}, \lambda=1.5 \mu \mathrm{m}$, and $\Delta \lambda=110 \mathrm{~nm}$ ). The collimated $\mathrm{THz}$ pulse is focused and the temporal characterization of its electric field is realized with the free space electro-optical sampling (EOS) technique [10] in a thin $\mathrm{GaP}$ crystal $[100 \mu \mathrm{m}$, (110) cut] [11] offering a flat electro-optical response over the emitted $\mathrm{THz}$ frequency range. The peak electric field magnitude is retrieved by the THz-induced intensity modulation of the sampling pulse [12].

An electric field as high as $1.5 \mathrm{MV} / \mathrm{cm} \pm 0.1 \mathrm{MV} / \mathrm{cm}$ is measured with the temporal field evolution presented in [Fig. 1(a), blue solid line]. The field exhibits one dominant cycle with a similar field strength for both polarities. The maximum field strength of $1.8 \mathrm{MV} / \mathrm{cm}$ could be achieved when shifting the absolute phase to zero [Fig. 1(a), black dashed]. The corresponding $\mathrm{THz}$ spectrum calculated via Fourier transformation [Fig. 1(b)] reflects a 5-octave spanning spectrum extending from 0.15 to $5.5 \mathrm{THz}$ with a central frequency of $f_{0}=\omega_{0} / 2 \pi=2.65 \mathrm{THz}$. We would like to emphasize that this frequency range has been challenging to access in the past at such high-field 

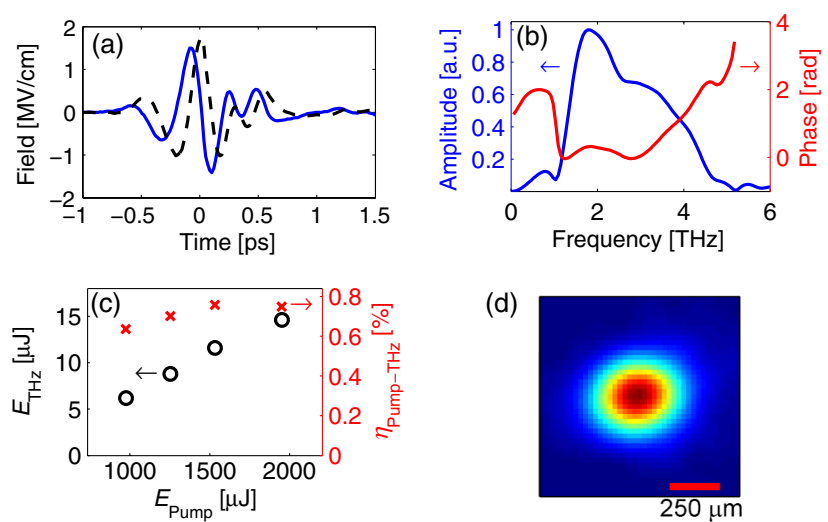

(d)

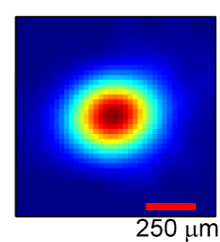

FIG. 1 (color online). (a) Field transient reconstructed by EOS (blue solid) and corresponding field shape for an absolute phase equal to zero (black dashed). The peak field reaches 1.5 and $1.8 \mathrm{MV} / \mathrm{cm}$, respectively. (b) Spectrum covering 5 octaves centered at $2.65 \mathrm{THz}$, with reconstructed spectral phase (red). (c) $\mathrm{THz}$ output energy and efficiency. (d) $\mathrm{THz}$ focal spot profile. Measurements are performed in dry air $(<0.5 \% \mathrm{RH})$.

strength since it is hardly accessible by optical rectification in $\mathrm{LiNbO}_{3}$ [6] (typically $<1 \mathrm{THz}$ ) and by difference frequency generation [13] $(>15 \mathrm{THz})$, respectively. The reconstructed spectrum carries the signature of phonon-induced absorption resonances in the DSTMS at $1 \mathrm{THz}, 2.7 \mathrm{THz}$, and above $5 \mathrm{THz}$, which in addition give rise to spectral phase nonlinearities [Fig. 1(b), red line]. The infrared to $\mathrm{THz}$ energy conversion efficiency is $\eta=$ $0.8 \%$ [Fig. 1(c)] and in line with other organic crystals $[12,14]$. It is limited by the onset of saturation. The peak pump fluence of $9 \mathrm{~mJ} / \mathrm{cm}^{2}$ is below the optical damage threshold of the crystal $\left(\approx 12 \mathrm{~mJ} / \mathrm{cm}^{2}\right)$ and gives rise to a $\mathrm{THz}$ energy of $15 \mu \mathrm{J}$ measured with a thermopile sensor placed in the vicinity of the focus. The focal spot size of $w_{0}=300 \mu \mathrm{m}\left(1 / e^{2}\right)$ shown in Fig. 1(d) is achieved by means of an $F / 2$-number off-axis parabola $(F=$ $101.6 \mathrm{~mm}$ ) and is recorded with a two-dimensional pyroelectric array detector. The waist is close to the diffraction limit $\left(270 \mu \mathrm{m}\right.$ at $\left.1 / e^{2}\right)$.

The intrinsic stability of the absolute phase for OR pulses allows us to monitor the evolution of the electric field [Fig. 1(a)] across the depth of focus $(\Delta z= \pm 6 \mathrm{~mm})$. A two-dimensional field measurement across the focus is shown in Fig. 2(a) together with the corresponding spectra (b). The Gouy effect changes significantly the electric field shape and gives rise to $\mathrm{a} \approx \pi$ phase shift for the transition from $z \ll-z_{R}$ to $z \gg z_{R}$, according to $\Phi=-\arctan \left(z / z_{R}\right)$ with $z_{R}$ being the Rayleigh range. Figure 2(a) illustrates that the local phase velocity of the electric field is increased along $z$ and unveils a superluminal-like phase change corresponding to a $200 \mathrm{fs}$ temporal advance of the electric field when compared to an equivalent but unfocused plane wave. Figure 2(b) illustrates the corresponding evolution in the spectral domain. The $\mathrm{THz}$ spectrum transforms from the off-focal, narrow band spectrum $(z=-6 \mathrm{~mm})$ towards
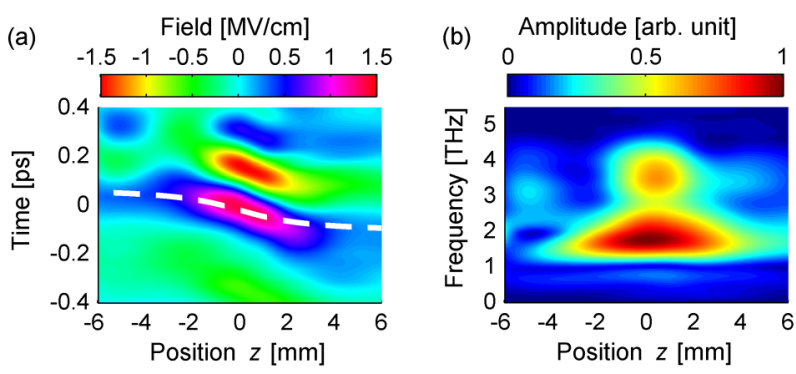

FIG. 2 (color online). (a) Temporal and (b) spectral evolution obtained by EOS across the focus at positions $z=$ $(-6,-4,-2,0,1,2,4,6) \mathrm{mm}$. The calculated Gouy phase shift for the central frequency $(2.65 \mathrm{THz}$, dashed line) deviates from the experimentally retrieved phase shift. The EOS pulse ( $\lambda=800 \mathrm{~nm}$, Rayleigh range $18 \mathrm{~mm}$ ) ensures an approximately constant probe area across the measurement range.

the 5-octave spanning supercontinuum $(z=0 \mathrm{~mm})$ and regains its narrowed shape behind the focus $(z=6 \mathrm{~mm})$. The evolution is due to the frequency-dependent geometrical focusing properties of Gaussian beams and the constant EO sampling volume. Out of focus $(z=6 \mathrm{~mm})$ lower frequency components (centered at $1.8 \mathrm{THz}$ ) are dominating the retrieved electric field while higher spectral components contribute significantly to field shape and phase properties in the waist $(z=0 \mathrm{~mm})$. The dashed line in Fig. 2(a) unveils that the Gouy phase shift for the central frequency $f_{0}$ does not accurately reflect the anomalous phase shift across the focus.

Apart from the Gouy phase, the instantaneous frequency is an additional pulse quantity whose exact knowledge is essential for field and frequency sensitive studies. The actual time variation of the electric field $E(t)$ is often represented as the product $E(t)=A(t) \cos \left(\omega_{0} t+\phi\right)$ with envelope $A(t)$, the (constant) frequency $\omega_{0}$, and the absolute phase $\phi$. This simple assumption becomes invalid for single-cycle multioctave spanning pulses simply because of violation of Maxwell's equations due to $\int_{-\infty}^{\infty} E d t \neq 0$ [15]. Here we confirm both experimentally and in simulation that tightly focused single-cycle pulses require indeed a more careful description.

More profound information on both the time and the frequency dynamics is gained by the WVDs [16] calculated at different locations across the focus. The WVD offers the best spectral resolution of all time-frequency representations since it does not rely on filter functions as required for short-time Fourier transformation. This distribution is thus suitable to investigate the evolution of focused singlecycle, supercontinuum carrying pulses. It furthermore offers direct access to the instantaneous frequency, chirp, and group delay. Shown in Fig. 3(c) are the retrieved WVDs for the field traces measured at different positions, and (d) summarizes the corresponding time-dependent instantaneous frequencies. The distribution unveils several important facts. First, the instantaneous frequency $f_{\text {inst }}(t)$ is not constant across the temporal pulse shape. In particular, at the beam waist $(z=0 \mathrm{~mm}) f_{\text {inst }}(t)$ carries a strong chirp 

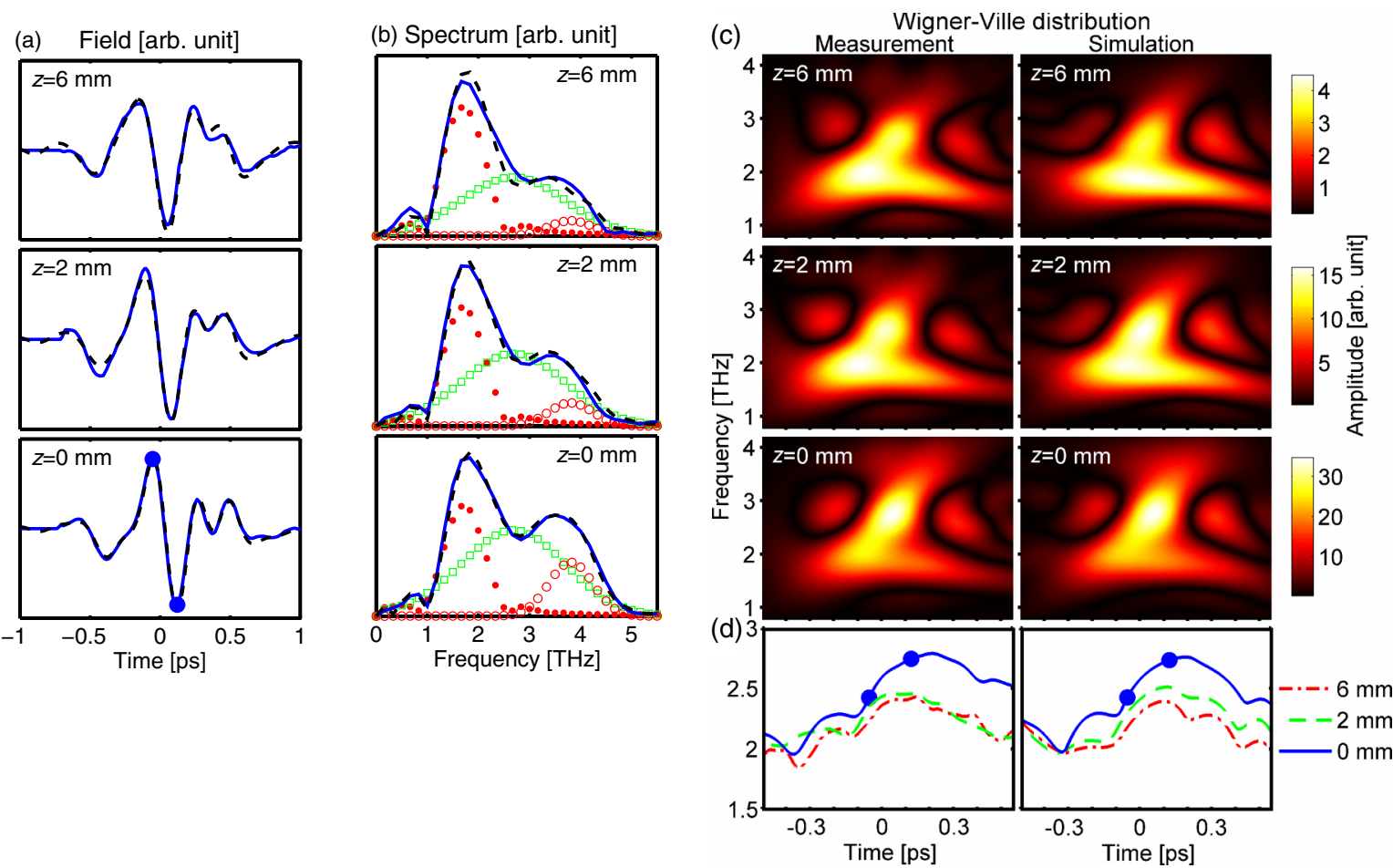

FIG. 3 (color online). (a) Normalized field reconstructed by EOS (blue solid line) and by our three-oscillator model (black dashed line) at different $z$. (b) Corresponding measured (blue solid line) and simulated (black dashed line) spectra. Spectra of the three oscillating dipoles used in our model are represented by red dots $(k=1)$, green squares $(k=2)$, and red circles $(k=3)$. (c) WignerVille distributions calculated from measured (left) and simulated (right) field traces. The color bar indicates the amplitude. In (d) the instantaneous frequencies are summarized. The blue dots indicate the instantaneous frequencies for the two opposing field peaks in (a) at the focus $z=0 \mathrm{~mm}$.

with a significant blueshift varying from $2 \mathrm{THz}$ at $-500 \mathrm{fs}$ to $2.8 \mathrm{THz}$ at $200 \mathrm{fs}$ after the main peak of the electric field. The instantaneous frequency for the corresponding positive and negative field peak [represented by blue dots in (a) and (d)] is thus significantly different $(2.4 \mathrm{THz}$ versus $2.7 \mathrm{THz})$, which is relevant for field and frequency sensitive applications. The observed self-induced intrapulse blueshift corresponds to an intrinsic temporal chirp and is a direct consequence of the Maxwell equations in vacuum for single-cycle pulses [17]. It is present in absence of any (non)linear pulse-matter interaction. Furthermore, our measurements unveil an additional positive chirp originating from the $\mathrm{THz}$ generation process itself and dispersion from $\mathrm{THz}$ optics which gives rise to the temporal asymmetry of $f_{\text {inst }}(t)$. Finally, we observe that the instantaneous frequency at maximum field depends strongly on the $z$ position. It drops from $2.7 \mathrm{THz}$ at the beam waist $(z=0 \mathrm{~mm})$ to $2.4 \mathrm{THz}$ behind the focus $(z=6 \mathrm{~mm})$.

The consequences of these findings for field-sensitive phenomena are eminent since knowledge of the precise form of the electric field and the absolute value of the instantaneous frequency in the focus are essential for an accurate description of the pulse-matter interaction (see, e.g., Ref. [7] for a review). It manifests the need of careful quantification of pulse properties in the focal waist by
WVD, for example, in particular for single-cycle pulses carrying multioctave spanning spectra. It furthermore corroborates the need of an appropriate model for pulse propagation which is presented next.

Our model for beam propagation is derived from recent work by Lin et al. [15]. Lin's complex-source-point model is based on an oscillating dipole (oscillator) and mimics a focused beam along the propagation axis $z$. We discovered that due to the modulated spectral shape of our $\mathrm{THz}$ pulses (Fig. 2) a single oscillator is not sufficient to describe accurately their propagation in $z$. We extend the simulation to three oscillators to account for the multiple octave spanning spectrum. Each oscillator is described by its dipole moment which is proportional to $p_{k}(t)$ (see Ref. [15] for more details) described by

$$
p_{k}(t)=p_{k} \exp \left(-\frac{t^{2}}{2 T_{k}^{2}}\right) e^{i \omega_{k} t+i \phi_{k}+i \Phi_{k}(t)}
$$

with the oscillator parameters $p_{k}$ amplitude, $T_{k}$ temporal width, $\omega_{k}$ the oscillator source frequency, $\phi_{k}$ phase offset, and $\Phi_{k}(t)$ the temporal phase. We restrict ourselves to a linear polarization in direction $x$, which is probed by EOS in our experiment. Each oscillator's field $E_{k}$ is propagated individually. For a given position in $z$ the 
fields of all oscillators on axis $(x=y=0)$ are summed up according to

$$
E(z, t)=\sum_{k=1}^{3} E_{k}(z, t)
$$

Hence the oscillators are coupled only by their electric field in the free space with the field $E_{k}(z, t)$ being the $x$ component of the simulated field. The model also describes the oscillator's fields for off-axis positions [15].

The oscillator parameters are chosen to best approximate the temporal $\mathrm{THz}$ field measured in the beam waist $(z=0 \mathrm{~mm})$, and consequently its Wigner-Ville distribution and the spectrum. The resulting oscillator frequencies are $f_{1}=1.6 \mathrm{THz}, f_{2}=2.2 \mathrm{THz}$, and $f_{3}=$ $3.7 \mathrm{THz}$. The individual oscillator pulse durations are $T_{k}=(0.85,0.53,1.35) 1 / f_{k}$. The absolute phase $\phi_{k}$ is chosen to reproduce the temporal pulse profile. For beam propagation, all oscillator parameters are kept constant for $z \neq 0$. The individual oscillator's spectra are shown in Fig. 3(b) as red, green, and red dashed lines. Because of the self-induced blueshift [15,17], the central frequencies are shifted up to $f_{k}^{\text {eff }}=(1.7,2.6,3.8) \mathrm{THz}$ in respect to the source oscillators $f_{k}$. They correspond to the center frequencies between the phonon resonances $(1.7$ and $3.8 \mathrm{THz})$ and to the central frequency $f_{0}(2.6 \mathrm{THz})$. The blueshift is caused by subcycle oscillator durations $T_{k}$. To represent the focusing dynamics when propagating through the focus, a collimated beam with nearly frequency-independent beam diameter is assumed at the focusing parabola. This is justified by our $\mathrm{THz}$ generation setup with collimated large diameter pump. The resulting numerical aperture defines the Rayleigh range for the individual oscillators [see $z_{0}$ [15] Eq. (3)].

The results of the three-oscillator model for $E(z, t)$ are summarized in Fig. 3 (dashed black line) and are compared to the experimentally retrieved electric field shape (a), spectrum (b), WVD (c), and instantaneous frequency (d). Simulations and measurements are shown for different longitudinal positions $z=(6,2,0) \mathrm{mm}$ before and at the focus and illustrate excellent agreement for all of the investigated pulse properties (a)-(d). It is worth noting that the simulation describes accurately the absolute amplitudes of both field and spectrum. The model matches the experimental data within an accuracy of $1 \% \mathrm{rms}$ and $3 \% \mathrm{rms}$ for the spectra and the temporal field shapes, respectively. These results underline the power of our multioscillator model to describe the propagation of multioctave spanning $\mathrm{THz}$ pulses across the focus.

An intriguing feature of single-cycle THz pulses is their potential to act as a quasiunipolar stimulus. The realization of such highly asymmetric electric field requires a control on the absolute phase. To our knowledge an absolute phase control for $\mathrm{THz}$ pulses has not been demonstrated so far. A change of the absolute phase requires the group velocity $v_{g}$ to be slightly varied compared to the

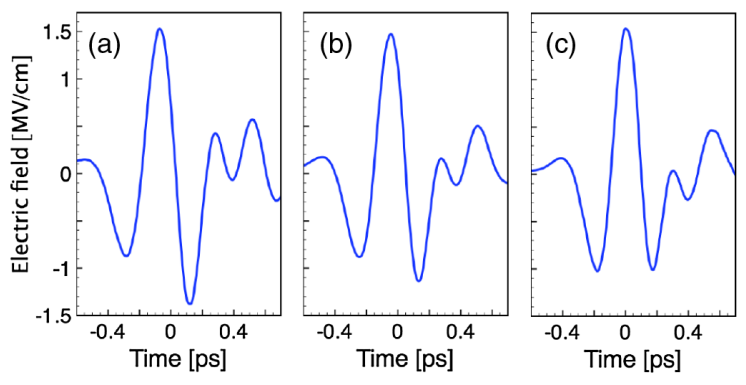

FIG. 4 (color online). Control of absolute phase at the focus with (a) $\pi / 3$, (b) $\pi / 6$, and (c) zero absolute phase achieved by dispersion control.

phase velocity $v_{\mathrm{ph}}$, according to $\Delta \phi=\omega d\left(1 / v_{\mathrm{ph}}-1 / v_{g}\right)$. The group velocity expressed by $v_{g}=\frac{c}{n}\left(1+\frac{\lambda}{n} \frac{\partial n}{\partial \lambda}\right)$ is introduced by wavelength-dependent delay with the help of a $\mathrm{THz}$ dispersive element. Here we propose to use a combination of $\mathrm{THz}$ transparent plastics, such as Teflon and TOPAS of variable thickness, to manipulate the absolute phase of the 5-octave spanning $\mathrm{THz}$ pulse. These materials introduce a group delay across the relevant $\mathrm{THz}$ range without inducing noticeable temporal pulse broadening and losses. In addition, Teflon acts as a low pass filter to separate the $\mathrm{THz}$ radiation from the infrared pump laser. With this technique the absolute phase can be controlled and set to zero to achieve the maximum field asymmetry in the waist. The results are shown in Fig. 4 for the field in the focus $(z=0 \mathrm{~mm})$ with three different sets of dispersive materials. With $2 \mathrm{~mm}$ of Teflon and $3 \mathrm{~mm}$ of TOPAS the maximum asymmetry could be realized exactly at the focal waist Fig. 4(c). An absolute phase shift of $(\pi / 6)$ and $(\pi / 3)$ are reached with the introduction of 2.8 and $5.2 \mathrm{~mm}$ Teflon without the TOPAS plate [Figs. 4(b) and 4(a)].

In conclusion, we showed that DSTMS is a well-suited material to generate multioctave spanning $\mathrm{THz}$ pulses ranging from 0.15 to $5.5 \mathrm{THz}$ with field strengths of $1.5 \mathrm{MV} / \mathrm{cm}$. The excellent $\mathrm{THz}$ beam wave front quality allowed tight focusing to a waist of $300 \mu \mathrm{m}$ close to the diffraction limited spot size. Our investigations visualized by Wigner-Ville distributions unveiled significant self-induced blueshifts and change of instantaneous frequency in the focus, which goes beyond the concept of the widely used carrier-envelope description assuming a constant frequency. These observations, which are relevant for field-sensitive investigations, were well reproduced by our multioscillator model describing the propagation of supercontinuum spanning pulses in the single-cycle regime. We finally presented direct control on the absolute phase enabling the formation of highly asymmetric, quasi-half-cycle pulses, which is a requirement for future high-field experiments.

We acknowledge support from SNSF (Grants No. PP00P2128493 and No. 200021122111). C.P.H. acknowledges association to NCCR-MUST. 
*clemens.ruchert@psi.ch

†christoph.hauri@psi.ch

[1] U. Fruhling, M. Wieland, M. Gensch, T. Gebert, B. Schutte, M. Krikunova, R. Kalms, F. Budzyn, O. Grimm, J. Rossbach, E. Plonjes, and M. Drescher, Nat. Photonics 3, 523 (2009).

[2] K. Kovacs, E. Balogh, J. Hebling, V. Tosa, and K. Varju, Phys. Rev. Lett. 108, 193903 (2012).

[3] A. Dienst, M. C. Hoffmann, D. Fausti, J.C. Petersen, S. Pyon, T. Takayama, H. Takagi, and A. Cavalleri, Nat. Photonics 5, 485 (2011).

[4] T. Kampfrath, A. Sell, G. Klatt, A. Pashkin, S. Mahrlein, T. Dekorsy, M. Wolf, M. Fiebig, A. Leitenstorfer, and R. Huber, Nat. Photonics 5, 31 (2011).

[5] J. Fulop, L. Palfalvi, M. Hoffmann, and J. Hebling, Opt. Express 19, 15090 (2011).

[6] H. Hirori, A. Doi, F. Blanchard, and K. Tanaka, Appl. Phys. Lett. 98, 091106 (2011).

[7] T. Brabec and F. Krausz, Rev. Mod. Phys. 72, 545 (2000).
[8] L. Mutter, F. D. Brunner, Z. Yang, M. Jazbinšek, and P. Günter, J. Opt. Soc. Am. B 24, 2556 (2007).

[9] M. Stillhart, A. Schneider, and P. Günter, J. Opt. Soc. Am. B 25, 1914 (2008).

[10] Q. Wu and X.-C. Zhang, Appl. Phys. Lett. 71, 1285 (1997).

[11] Q. Wu and X.-C. Zhang, Appl. Phys. Lett. 70, 1784 (1997).

[12] C. Ruchert, C. Vicario, and C. P. Hauri, Opt. Lett. 37, 899 (2012).

[13] A. Sell, A. Leitenstorfer, and R. Huber, Opt. Lett. 33, 2767 (2008).

[14] C. P. Hauri, C. Ruchert, C. Vicario, and F. Ardana, Appl. Phys. Lett. 99, 161116 (2011).

[15] Q. Lin, J. Zheng, and W. Becker, Phys. Rev. Lett. 97, 253902 (2006).

[16] L. Coen, Time-Frequency Analysis (Prentice-Hall, New York, 1995).

[17] Q. Lin, J. Zheng, J. Dai, I. Chen Ho, and X.-C. Zhang, Phys. Rev. A 81, 043821 (2010). 